\title{
Los proyectos de aprendizaje tutorado como buena práctica en educación física en primer curso de doble titulación (educación infantil y educación primaria)
}

\author{
Tutored learning projects as a good practice in physical \\ education in the first course of double grade (childhood \\ education and primary education)
}

\author{
Miriam Molina Soria ${ }^{1}$, Víctor M. López Pastor ${ }^{2}$, Cristina Pascual Arias $^{3}$ \& Raúl \\ Alberto Barba Martín ${ }^{4}$
}

Fecha de recepción: 25/01/2019; Fecha de revisión: 22/02/2019; Fecha de aceptación: 22/03/2019

Cómo citar este artículo:

Molina Soria, M., López-Pastor, V. M., Pascual Arias, C., \& Barba Martín, R. A. (2019). Los proyectos de aprendizaje tutorado como buena práctica en educación física en primer curso de doble titulación (educación infantil y educación primaria). Revista de Innovación y Buenas Prácticas Docentes, 8, 1-14.

\section{Autor de Correspondencia: miriam.molina@uva.es}

\section{Resumen:}

En este trabajo presentamos los resultados de una buena práctica llevada a cabo en la asignatura de fundamentos de la educación física en educación infantil: Ios "Proyectos de Aprendizaje tutorado".

En la primera parte se describe la organización de la asignatura y el ejemplo de buena práctica. En la segunda parte se presentan los resultados obtenidos, que son muy positivos.

Hay un cambio relevante en la carga de trabajo del profesor, que puede explicarse por los años de experiencia del profesorado que desarrolla la asignatura. Se refleja en conclusiones.

Palabras clave: Educación Física, Educación Infantil, Proyecto de Trabajo Tutorado, Evaluación formativa.

\section{Abstract:}

In this paper we present the results of a good practice carried out in the subject of foundations of Physical Education in Early Childhood Education: the "Tutored Learning Projects". The first part describes the organization of the subject and the example of good practice. In the second part the results obtained are presented, which are very positive. There is a relevant change in the workload of the professor, which can be explained by the years of experience of the teaching staff that develops the subject. It is reflected in conclusions.

Key Words: Physical Education, Early Chilhood Education, Tutored Learning Projects, Formative Assessment

\footnotetext{
1 Universidad de Valladolid (España), miriam.molina @uva.es; CÓDIGO ORCID: 0000-0003-2974-5535

2 Universidad de Valla dolid (España), vlopez@mpc.uva.es; CÓDIGO ORCID: 0000-0003-2681-9543

3 Universidad de Valladolid (España), cristina.pascual@uva.es; CÓDIGO ORCID: 0000-0002-2781-5600 ${ }^{4}$ Universidad de Valla dolid (España), raula lberto.brba@uva.es; CÓDIGO ORCID: https://orcid.org/0000-00030071-687X
} 


\section{INTRODUCCIÓN}

Este artículo analiza una experiencia de evaluación formativa y compartida en la titulación de doble grado de educación infantil y educación primaria durante un cuatrimestre. La asignatura, fundamentos y didáctica de la educación corporal en educación infantil, es de seis créditos totales, que se reparten en prácticas, seminarios y teoría.

El artículo se organiza en tres grandes apartados: desarrollo de la experiencia de innovación, resultados y conclusiones.

\section{DESARROLLO DE LA EXPERIENCIA DE INNOVACIÓN}

En el centro llevamos muchos años trabajando con los Proyectos de Aprendizaje Tutorado (PAT), porque han demostrado ser una forma muy eficaz de trabajo teóricopráctico y de desarrollo de competencias profesionales. Se trata de una actividad de aprendizaje grupal. Cada grupo tiene que preparar una sesión práctica y un marco teórico a elegir dentro de una lista de temas y propuestas de intervención que se ofrecen a la clase a principio de curso. Se asigna una fecha de realización para cada PAT. En tutorías se explica a cada grupo los documentos que tienen que leer sobre cada propuesta y deben realizar una serie de tutorías obligatorias con los borradores de sus PAT antes de poder llevarlo a cabo, de forma que se asegura una calidad mínimamente aceptable en la puesta en práctica y en la documentación a entregar a sus compañeras (un marco teórico de 4 páginas).

\subsection{Resumen de los sistemas de evaluación de la asignatura}

En la tabla 1 se indican el total de alumnos matriculados en la asignatura y los tipos de sistemas de evaluación y calificación a poder elegir por el alumnado.

Tabla 1.

Sistemas de evaluación de la asignatura.

\begin{tabular}{|c|c|c|c|}
\hline Sistema & A-Continua & B- Mixta & C-Final \\
\hline Alumno/as matriculados & \multicolumn{3}{|c|}{47} \\
\hline NNo alumno/as por opción & 26 & 16 & 4 \\
\hline $\begin{array}{c}\text { Evaluación inicial o } \\
\text { diagnóstica }\end{array}$ & & & \\
\hline Requisitos & $\begin{array}{l}\text { Señalados en } \\
\text { texto }\end{array}$ & $\begin{array}{c}\text { Asistencia 50\% } \\
+ \text { Pat }\end{array}$ & nada \\
\hline Actividades de aprendizaje & Todas & $\begin{array}{l}\text { Solo PAT + } \\
\text { voluntario }\end{array}$ & $\begin{array}{l}\text { Exámenes finales ( } 3 \\
\text { pruebas diferentes) }\end{array}$ \\
\hline
\end{tabular}

Como puede comprobarse existen tres vías para poder cursar la asignatura. Cada alumno elige cual prefiere seguir, según sus circunstancias y preferencias. Cada vía tiene unos requisitos, explicados en la tabla. Los requisitos mínimos establecidos para poder trabajar en la "vía continua" son los siguientes:

a) asistencia regular a clase, justificando cada falta y con un máximo del $20 \%$.

b) entrega de todos los documentos en tiempo y forma y corrección de los que estén mal dentro del plazo establecido (una semana).

c) asistencia a todas las tutorías que sean necesarias para garantizar una calidad mínima en el PAT antes de llevarse a cabo con las compañeras. 


\subsection{Resumen de las actividades de evaluación y calificación de la asignatura, vía de "evaluación continua"}

En la tabla 2 presentamos un resumen de las actividades de evaluación y calificación que forman el sistema básico de trabajo en la vía de evaluación continua.

Tabla 2.

Resumen de las actividades de evaluación y calificación.

\begin{tabular}{llc}
\hline \multicolumn{2}{c}{ Evaluación } & Calificación \\
\hline \multicolumn{1}{c}{ Actividades de aprendizaje } & \multicolumn{1}{c}{ Instrumento } & $\%$ \\
\hline 1.PAT & Escala descriptiva (rúbrica) & 30 \\
\hline $\begin{array}{l}\text { 2.Trabajo grupal sobre el currículum de El } \\
\text { y recensiones sobre lecturas }\end{array}$ & Escala descriptiva & 20 \\
\hline $\begin{array}{l}\text { 3.Fichas de sesiones prácticas } \\
\text { 4.Examen parcial con co-evaluación } \\
\text { inmediata }\end{array}$ & Elantilla de corrección "ad hoc" & 20 \\
\hline \begin{tabular}{l} 
5.Mapas conceptuales \\
\hline
\end{tabular} & Escala descriptiva (rúbrica) & 10 \\
\hline
\end{tabular}

\subsection{Actividad de aprendizaje y evaluación: Proyecto de Aprendizaje Tutorado}

Se trata de una actividad de aprendizaje grupal. Cada grupo tiene que preparar una sesión práctica y un marco teórico a elegir dentro de una lista de temas y propuestas de intervención que se ofrecen a la clase a principio de curso. El proceso de elaboración es el siguiente:

a) Se asigna una fecha de realización para cada PAT.

b) En tutorías se explica a cada grupo los documentos que tienen que leer sobre cada propuesta.

c) El grupo elabora un borrador del marco teórico (4 páginas) y un plan de sesión y acuden a una tutoría grupal, donde el profesor corrige los borradores y les dice las cosas que deben ser mejoradas y corregidas.

d) Este proceso se repite hasta que los dos documentos tienen una calidad aceptable.

e) Se realiza la sesión práctica en la fecha elegida y a continuación se expone el marco teórico en 10 minutos, entregando previamente una copia en papel del mismo a cada compañera-0.

f) Una semana después deben entregar el informe final del PAT. Si está correcto el profesor se lo devuelve para que lo guarden en su carpeta. Si hay aspectos a mejorar deben corregirlo y volverlo a entregar en un plazo máximo de una semana.

Además, debemos tener en cuenta las cuestiones necesarias a tener en cuenta para que la aplicación sea eficaz:

a) Establecer de forma clara el procedimiento a seguir.

b) Asistencia obligatoria de todo el grupo a todas las tutorías que sean necesarias para garantizar una calidad mínima en el PAT antes de llevarse a cabo con las compañeras.

c) Facilitar instrumentos de autoevaluación sobre la calidad del PAT.

d) Dar suficiente feedback durante el proceso para garantizar la calidad final del PAT cuando se vaya a presentar ante las compañeras. 
Tal como se ha demostrado en nuestro centro en los últimos 15 años, se trata de una metodología muy eficaz. En muchas asignaturas el PAT se lleva a cabo con alumnado de infantil o primaria en horario lectivo real, en algunos de los centros educativos de la ciudad. Suele ser más interesante para el alumnado, pero mucho más complejo de organizar para el profesorado, especialmente cuando el número de alumnos y grupos es tan elevado como en esta asignatura. Pueden encontrarse algunos artículos publicados en que se explica a fondo estas experiencias y su organización (Manrique, López, Monjas \& Real, 2010; Barba, López, Manrique, Gea \& Monjas, 2010; López, Manrique, Monjas \& Gea, 2010).

Esta actividad concreta de aprendizaje requiere varios instrumentos que permitan valorar el proceso o los resultados:

a) Fichas de autoevaluación para el PAT.

b) Escalas descriptivas para la evaluación y la calificación del PAT.

c) Rúbrica específica de autoevaluación del PAT.

d) Ficha de observación de la presentación oral del PAT del grupo y de cada componente.

\section{RESULTADOS}

Al finalizar la asignatura se aplican diferentes instrumentos de evaluación anónima a los estudiantes. El primero es un cuestionario estructurado para evaluar la percepción de ventajas e inconvenientes del alumnado, tanto de la "buena práctica" (en este caso, el PAT), como de la asignatura completa. El proceso de validación científica del cuestionario puede encontrarse en Castejón-Oliva, Santos-Pastor, \& Palacios (2015). A continuación presentamos los resultados obtenidos.

\subsection{Cuestiones preguntadas al alumnado sobre la experiencia. Cuestionario sobre la buena práctica}

En la tabla 3 se incluyen los resultados del alumnado encuestado sobre la "buena práctica" desarrollada, que en este caso es el PAT.

Tabla 3.

Resultados de los cuestionarios sobre la buena práctica.

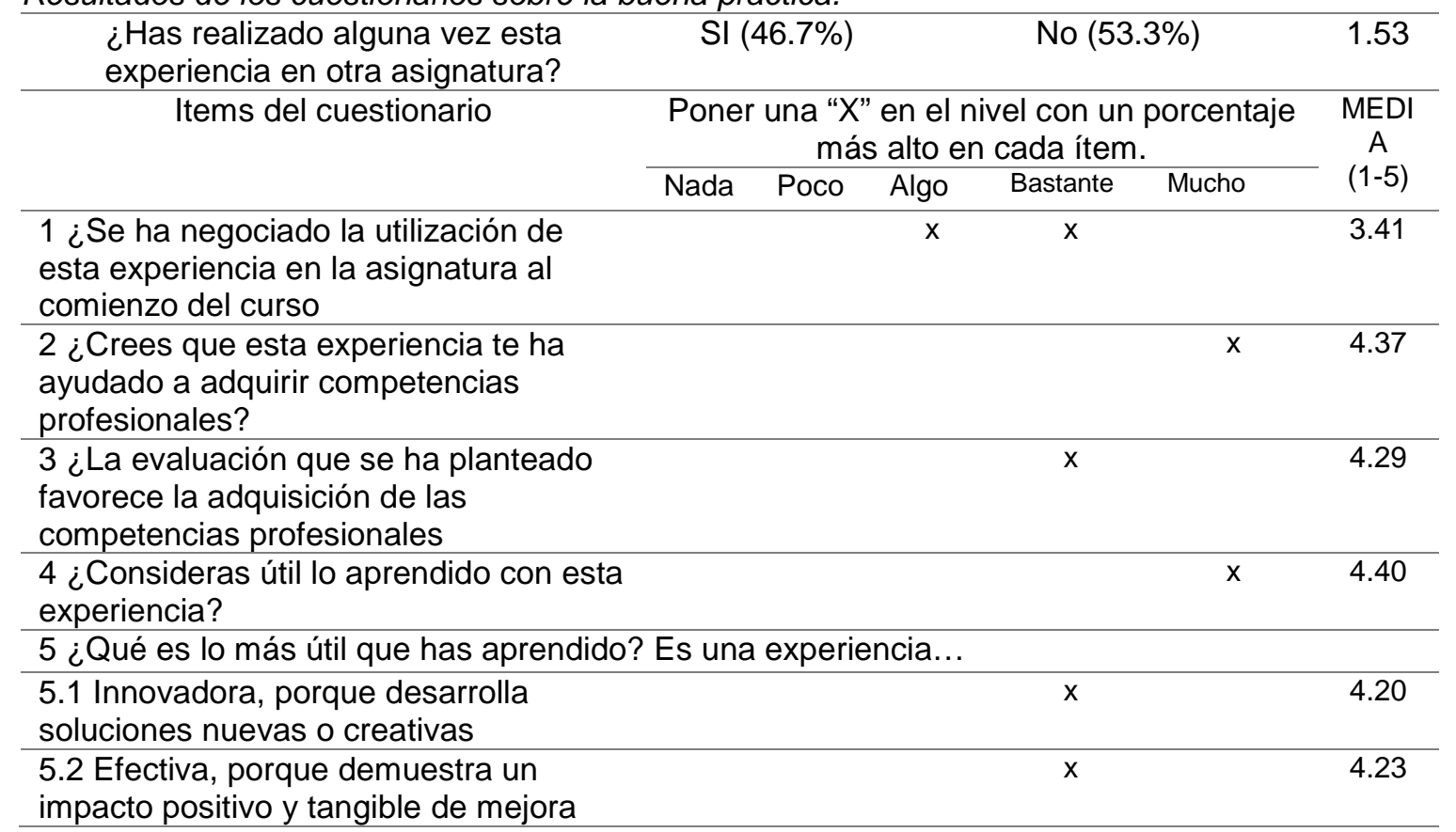


Tabla 3.

Resultados de los cuestionarios sobre la buena práctica (continuación).

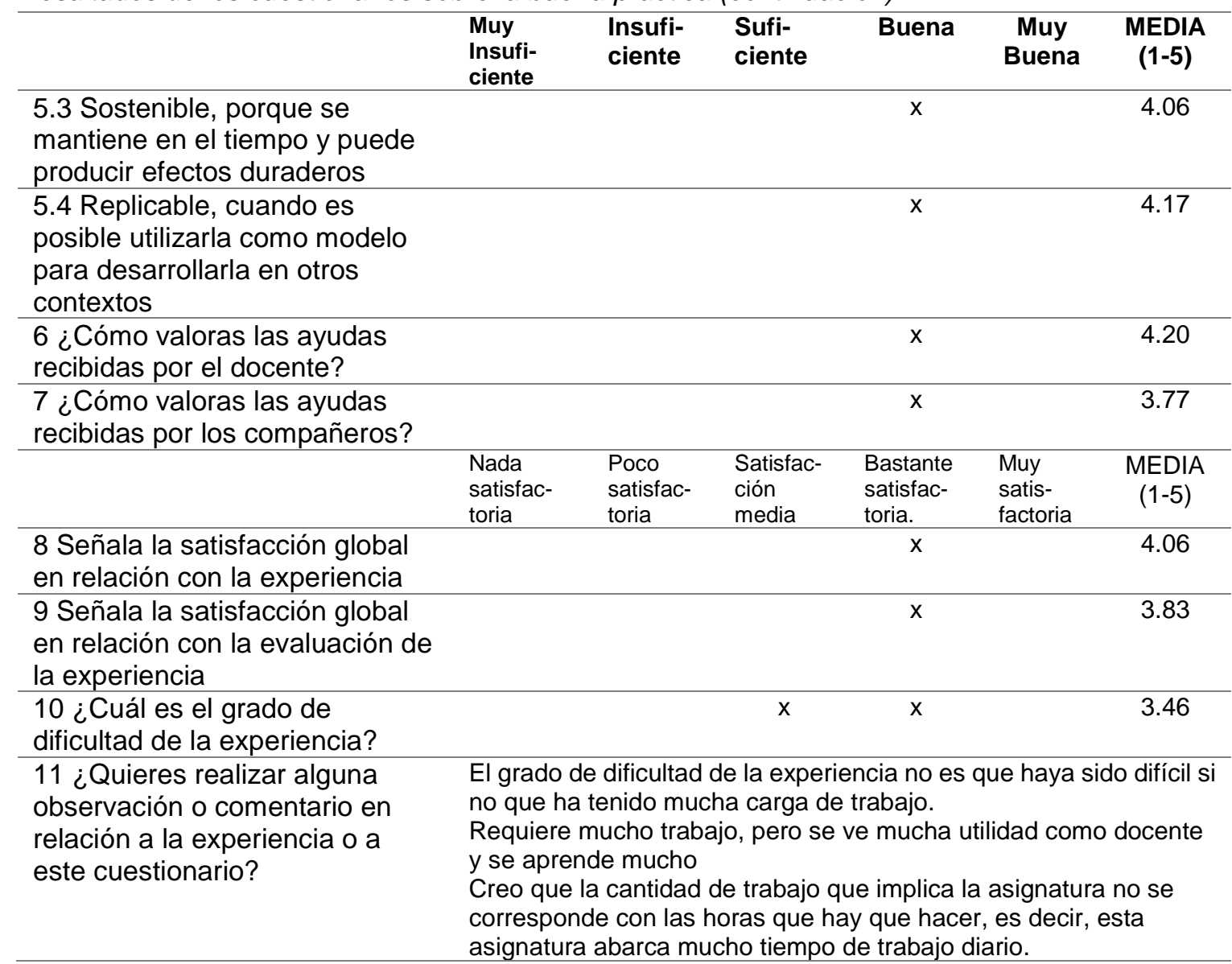

Como puede comprobarse, las valoraciones son muy altas en la mayoría de los ítems, por lo que la experiencia de buena práctica tiene una buena acogida y satisfacción en el alumnado.

Asimismo, como docente se realiza una valoración de la experiencia de buena práctica. Los docentes que imparten la asignatura consideran que esta experiencia es:

a) Innovadora; al desarrollar soluciones nuevas o creativas, porque supone una gran diferencia con modelos más tradicionales de enseñanza. Es una metodología activa y exige una fuerte colaboración grupal para que salga bien.

b) Efectiva; cuando demuestra un impacto positivo y tangible de mejora, porque al ser obligatoria la realización de tutorías durante la elaboración del proyecto, la calidad del mismo mejora considerablemente.

c) Sostenible; al mantenerse en el tiempo y producir efectos duraderos, porque supone una mayor carga de trabajo en las tutorías de los meses centrales, cuando la mayoría de los grupos están elaborando su PAT y requieren tutorías, pero es perfectamente viable, dado que se realiza en las horas oficiales de tutoría.

d) Replicable; cuando es posible utilizarla como modelo para desarrollarla en otros contextos, porque se puede utilizar en cualquier asignatura. De hecho, 
en nuestro centro es una metodología muy utilizada desde hace 15-20 años en muchas asignaturas.

Como valoración global, los docentes que imparten la asignatura consideran que se trata de una actividad de aprendizaje que funciona muy bien, con una tasa de éxito muy elevada y con una buena calidad general. La explicación de ello está en la obligatoriedad y sistematicidad del proceso de revisión antes de poder poner en práctica el PAT. A pesar de ello este año ha habido problemas con varios PAT en este grupo. Son de primer curso (doble titulación de infantil y primaria) y ha habido un porcentaje muy elevado de alumnos que se han ido descolgando y funcionando mal. Casi un $50 \%$ del grupo ha terminado pasando a la vía mixta por no haber funcionando correctamente durante la asignatura. Hemos observado mucho "efecto polizón", muchas personas que no aportaban nada al grupo, solo se dejan llevar. Esto ha generado varios conflictos en junio, cuando llega la hora de repartir las calificaciones dentro de cada grupo.

La perspectiva del alumnado con respecto a la experiencia es: (a) mucho trabajo para poco tiempo; (b) al ser cuatro profesores hubo veces que unos tenían un criterio de corrección diferente que otros; (c) demasiados trabajos para realizar en casa; no deja mucho tiempo a otras asignaturas.

\subsubsection{Ventajas e inconvenientes encontradas. Propósitos de cambios para el próximo curso}

Como ventajas podemos señalar la tasa de éxito elevada, a pesar de la baja implicación de algunos alumnos; la buena calidad en muchos de los PAT realizados; y la buena preparación en gran parte de los grupos, pero mediocres y flojos otros.

Los principales problemas encontrados han sido que algunos grupos que preparan el trabajo de forma deficiente, y es complicado evitarlo a pesar de las tutorías grupales. Son pocos casos y la mayoría del alumnado ha realizado bien su trabajo.

Como propósitos de cambio para el próximo curso señalamos la utilización de forma sistemática de la plantilla de autoevaluación y autocalificación del PAT que ya se empleó el año anterior y ofrece una orientación importante al alumnado.

\subsection{Aspectos relacionados con el cuestionario y en relación a la asignatura a nivel general}

En las figuras 1 y 2 , se detallan las ventajas e inconvenientes que considera el alumnado sobre la evaluación de la asignatura. La X muestra la opción elegida por un mayor porcentaje de alumnado para cada uno de los ítems.

\begin{tabular}{|c|c|c|c|c|c|}
\hline \multirow{2}{*}{$\begin{array}{l}\text { Aspectos que se consideran por parte del } \\
\text { alumnado }\end{array}$} & \multicolumn{5}{|c|}{$\begin{array}{c}\text { Poner una "X" en el nivel con un porcentaje más } \\
\text { alto en cada ítem. }\end{array}$} \\
\hline & Nada & Poco & Algo & Bastante & Mucho \\
\hline Ofrece alternativas a todos los estudiantes & & & & $\mathrm{X}$ & \\
\hline $\begin{array}{l}\text { Hay un contrato previo, negociado y } \\
\text { consensuado del sistema de evaluación }\end{array}$ & & & & $\mathrm{X}$ & \\
\hline $\begin{array}{l}\text { Está centrado en el proceso, importancia del } \\
\text { trabajo diario }\end{array}$ & & & & & $\mathrm{X}$ \\
\hline El estudiante realiza un aprendizaje activo & & & & & $\mathrm{X}$ \\
\hline $\begin{array}{l}\text { Se plantea el trabajo en equipo de forma } \\
\text { colaborativa }\end{array}$ & & & & & $\mathrm{X}$ \\
\hline $\begin{array}{l}\text { El alumno/a está más motivado, el proceso } \\
\text { de aprendizaje es más motivador }\end{array}$ & & & $\mathrm{X}$ & & \\
\hline La calificación es más justa & & & & $\mathrm{X}$ & \\
\hline $\begin{array}{l}\text { Mejora la tutela académica (seguimiento y } \\
\text { ayuda al alumno/a) }\end{array}$ & & & & $\mathrm{X}$ & \\
\hline
\end{tabular}




\begin{tabular}{|c|c|c|c|c|c|}
\hline \multirow{2}{*}{$\begin{array}{l}\text { Aspectos que se consideran por parte del } \\
\text { alumnado }\end{array}$} & \multicolumn{5}{|c|}{$\begin{array}{c}\text { Poner una "X" en el nivel con un porcentaje más } \\
\text { alto en cada ítem. }\end{array}$} \\
\hline & Nada & Poco & Algo & Bastante & Mucho \\
\hline Permite aprendizajes funcionales & & & & $\mathrm{X}$ & \\
\hline Genera aprendizajes significativos & & & & $x$ & \\
\hline Se aprende mucho más & & & & $\mathrm{X}$ & \\
\hline Mejora la calidad de los trabajos exigidos & & & & $\mathrm{X}$ & \\
\hline Hay interrelación entre teoría y práctica & & & & & X \\
\hline $\begin{array}{l}\text { Evalúa todos los aspectos posibles (en } \\
\text { referencia al saber, saber hacer y saber } \\
\text { estar y ser) }\end{array}$ & & & & $\mathrm{X}$ & \\
\hline \multicolumn{6}{|c|}{ Figura 1. Ventajas que se reconocen en el Sistema de evaluación aplicado (continuación). } \\
\hline \multirow{2}{*}{$\begin{array}{l}\text { Aspectos que se consideran por parte del } \\
\text { alumnado }\end{array}$} & \multicolumn{5}{|c|}{$\begin{array}{c}\text { Poner una "X" en el nivel con un porcentaje más } \\
\text { alto en cada ítem. }\end{array}$} \\
\hline & Nada & Poco & Algo & Bastante & Mucho \\
\hline $\begin{array}{l}\text { Hay retroalimentación en documentos y } \\
\text { actividades }\end{array}$ & & & & & $X$ \\
\hline $\begin{array}{l}\text { Hay posibilidad de corregir errores en } \\
\text { documentos y actividades }\end{array}$ & & & & & $\mathrm{X}$ \\
\hline Se da un seguimiento más individualizado & & & & $\mathrm{X}$ & \\
\hline Requiere más responsabilidad & & & & & X \\
\hline
\end{tabular}

Figura 1. Ventajas que se reconocen en el Sistema de evaluación aplicado (continuación).

La figura 2 muestra la valoración sobre los posibles inconvenientes que marca el cuestionario.

\begin{tabular}{|c|c|c|c|c|c|}
\hline \multirow{2}{*}{$\begin{array}{l}\text { Aspectos que se consideran por parte del } \\
\text { alumnado }\end{array}$} & \multicolumn{5}{|c|}{$\begin{array}{c}\text { Poner una "X" en el nivel con un porcentaje } \\
\text { más alto en cada ítem. }\end{array}$} \\
\hline & Nada & Poco & Algo & Bastante & Mucho \\
\hline 1. Exige una asistencia obligatoria y activa & & & & & $X$ \\
\hline $\begin{array}{l}\text { 2. Tiene una dinámica de trabajo poco conocida, } \\
\text { falta de hábito }\end{array}$ & & & & $\mathrm{X}$ & \\
\hline 3. Exige continuidad & & & & & $x$ \\
\hline 4. Hay que comprenderlo previamente & & & & $\mathrm{X}$ & \\
\hline 5. Exige un mayor esfuerzo & & & & & $X$ \\
\hline 6. Existe dificultad para trabajar en grupo & & & $\mathrm{X}$ & & \\
\hline 7. Se puede acumular mucho trabajo al final & & & & & $X$ \\
\hline 8. Existe una desproporción trabajo/créditos & & & & & $X$ \\
\hline $\begin{array}{l}\text { 9. El proceso de calificación es más complejo y, a } \\
\text { veces, poco claro }\end{array}$ & & $\mathrm{x}$ & & & \\
\hline $\begin{array}{l}\text { 10. Genera inseguridad e incertidumbre, dudas } \\
\text { sobre que hay que realizar }\end{array}$ & & $\mathrm{X}$ & & & \\
\hline 11. Es injusto frente a otros procesos de evaluación & $\mathrm{X}$ & & & & \\
\hline 12. Las correcciones han sido poco claras & $\mathrm{x}$ & & & & \\
\hline 13. La valoración del trabajo es subjetiva & & & $\mathrm{X}$ & & \\
\hline $\begin{array}{l}\text { 14. Exige participar en mi propia evaluación } \\
\text { (autoevaluarse) }\end{array}$ & & & & & $X$ \\
\hline
\end{tabular}

Figura 2. Inconvenientes que se reconocen en el Sistema de evaluación aplicado.

Respecto a los aspectos que se consideran por parte del profesorado, debemos tener en cuenta que los docentes están bastantes satisfechos con el sistema de 
evaluación utilizado, aunque están bastante de acuerdo con que supone una carga de trabajo excesiva y algo de acuerdo con el alumnado presenta resistencias frente al sistema de evaluación continua y formativa.

\subsubsection{Datos del cuestionario de autopercepción de competencias}

En la tabla 4 se organizan los datos del cuestionario de autopercepción de competencia de los estudiantes al principio y al final de la asignatura.

Se trata del segundo cuestionario que se trabaja con el alumnado. El cuestionario se centra en la percepción de competencia docente y se divide en tres escalas: competencias transversales, competencias docentes competencias específicas docentes de educación física.

El proceso de validación científica del cuestionario puede encontrarse en Salcines, González-Fernández, Ramírez-García, \& Martínez-Mínguez (2018).

Los resultados se ofrecen en dos bloques de dos columnas cada uno: (1) media y desviación típica (DT) en el ítem del pretest (principio asignatura); (2) media y DT en los ítems del postest (al final de la asignatura) (ver tabla 4).

Tabla 4.

Resultados del bloque 1 del cuestionario de autopercepción de competencias.

\begin{tabular}{|c|c|c|c|c|}
\hline \multicolumn{5}{|c|}{ Bloque I. Competencias transversales } \\
\hline \multirow[b]{2}{*}{ Items } & \multicolumn{2}{|c|}{$\begin{array}{l}\text { Principio } \\
\text { asignatura }\end{array}$} & \multicolumn{2}{|c|}{$\begin{array}{c}\text { Final } \\
\text { Asignatura }\end{array}$} \\
\hline & Media & DT & Media & DT \\
\hline 1.1 Analizar y sintetizar & 2.97 & .560 & 3 & .508 \\
\hline 1.2 Organizar y planificar & 3.25 & .554 & 3.31 & .693 \\
\hline 1.3 Comunicarse de forma oral y escrita en lengua nativa & 3.36 & .639 & 3.19 & .592 \\
\hline 1.4 Comunicarse gestual y corporalmente & 3.19 & .668 & 3.13 & .554 \\
\hline 1.5 Conocer una lengua extranjera & 2.79 & .808 & 2.50 & .842 \\
\hline $\begin{array}{l}\text { 1.6 Utilizar las Tecnologías de la Información y la } \\
\text { Comunicación en el ámbito de estudio }\end{array}$ & 3.06 & .684 & 3.03 & 695 \\
\hline 1.7 Trabajar en equipo & 3.42 & 692 & 3.47 & 621 \\
\hline $\begin{array}{l}\text { 1.8 Desarrollar habilidades en las relaciones } \\
\text { interpersonales (empatía, asertividad, respeto y } \\
\text { escucha) }\end{array}$ & 3.70 & .467 & 3.63 & .554 \\
\hline $\begin{array}{l}\text { 1.9 Desarrollar habilidades intrapersonales (autoestima, } \\
\text { motivación y confianza en uno mismo) }\end{array}$ & 3 & .676 & 3.28 & .729 \\
\hline 1.10 Razonar de forma crítica y reflexiva & 2.94 & .715 & 3.16 & .574 \\
\hline 1.11 Desarrollar un compromiso ético & 3.22 & .540 & 3.22 & .706 \\
\hline 1.12 Desarrollar un aprendizaje autónomo & 3.08 & .649 & 3.19 & .644 \\
\hline 1.13 Adaptarse a situaciones nuevas & 3.25 & .692 & 3.28 & .634 \\
\hline 1.14 Desarrollar la creatividad & 3 & .926 & 3.28 & .772 \\
\hline
\end{tabular}

Como se puede observar, todos los ítems aumentan levemente su media aritmética cuando se pasa el cuestionario al final de la asignatura (post test), excepto comunicarse de forma oral y escrita en lengua nativa, comunicarse gestual y corporalmente, conocer una lengua extranjera, utilizar las tecnologías de la información y la comunicación en el ámbito de estudio, y desarrollar habilidades en las relaciones interpersonales, que experimentan una media ligeramente inferior en el postest que en el pretest.

El ítem de razonar de forma crítica y reflexiva no ha experimentado ningún cambio en la media aritmética al final de la asignatura. 
Tabla 5.

Resultados del bloque 2 del cuestionario de autopercepción de competencias.

\begin{tabular}{|c|c|c|c|c|}
\hline \multicolumn{5}{|c|}{ Bloque II: Competencias docentes } \\
\hline & \multicolumn{2}{|c|}{$\begin{array}{l}\text { Principio } \\
\text { asignatura }\end{array}$} & \multicolumn{2}{|c|}{$\begin{array}{c}\text { Final } \\
\text { Asignatura }\end{array}$} \\
\hline & Media & DT & Media & DT \\
\hline $\begin{array}{l}\text { 2.1. Conocer las características organizativas de los } \\
\text { centros educativos }\end{array}$ & 2.42 & .500 & 2.78 & .553 \\
\hline $\begin{array}{l}\text { 2.2. Elaborar propuestas de cambio de la realidad } \\
\text { educativa }\end{array}$ & 2.74 & .701 & 2.91 & 689 \\
\hline 2.3. Diseñar situaciones de aprendizaje & 2.86 & .683 & 3.13 & .492 \\
\hline 2.4. Animar situaciones de aprendizaje & 3.17 & .507 & 3.13 & .562 \\
\hline 2.5. Gestionar la progresión de los aprendizajes & 2.66 & .591 & 2.81 & .535 \\
\hline 2.6. Diseñar estrategias de atención a la diversidad & 2.67 & .717 & 3.16 & 677 \\
\hline $\begin{array}{l}\text { 2.7. Poner en práctica estrategias de atención a la } \\
\text { diversidad }\end{array}$ & 2.83 & .737 & 2.81 & .896 \\
\hline 2.8. Implicar al alumnado en su aprendizaje & 3.22 & .637 & 3.09 & .530 \\
\hline 2.9. Implicar al alumnado en la vida del centro & 3.06 & .791 & 3 & .568 \\
\hline 2.10. Participar en la gestión del Centro & 2.37 & .808 & 2.56 & .716 \\
\hline 2.11. Informar a las familias sobre la evolución de su hijo/a & 3.18 & .626 & 2.97 & .816 \\
\hline 2.12. Implicar a las familias en el aprendizaje de su hijo/a & 3.14 & .639 & 2.88 & .793 \\
\hline 2.13. Implicar a las familias en la vida del centro & 3 & .717 & 2.91 & .588 \\
\hline 2.14. Afrontar los deberes y dilemas éticos de la profesión & 3.06 & .765 & 2.94 & .680 \\
\hline $\begin{array}{l}\text { 2.15. Atender al propio desarrollo profesional docente } \\
\text { (autoevaluación, lecturas, cursos, jornadas, congresos, } \\
\text { redes de trabajo) }\end{array}$ & 2.86 & .723 & 2.88 & .609 \\
\hline $\begin{array}{l}\text { 2.16. Desarrollar procesos de innovación educativa en el } \\
\text { aula }\end{array}$ & 2.92 & .732 & 2.91 & 641 \\
\hline $\begin{array}{l}\text { 2.17. Poner en marcha procesos de investigación en el } \\
\text { aula }\end{array}$ & 2.67 & .759 & 2.84 & .677 \\
\hline
\end{tabular}

Respecto a la tabla 5, muchos ítems aumentan su media aritmética del pretest al postest. Aunque nos gustaría señalar algunos ítems que sufren una ligera bajada de la media al final de la asignatura. Estos ítems están relacionados con la colaboración de las familias en la escuela; los ítems que sufren dichos cambios son: implicar al alumnado en su aprendizaje, implicar al alumnado en la vida del centro, informar a las familias sobre la evolución de su hijo/a, implicar a las familias en el aprendizaje de su hijo/a, e implicar a las familias en la vida del centro.

Otros ítems que hay sufrido una leve bajada de la media han sido: animar situaciones de aprendizaje, diseñar estrategias de atención a la diversidad, poner en práctica estrategias de atención a la diversidad y desarrollar procesos de innovación educativa. 
Tabla 6.

Resultados del bloque 3 del cuestionario de autopercepción de competencias.

\begin{tabular}{|c|c|c|c|c|}
\hline \multicolumn{5}{|c|}{ Bloque III: Competencias específicas docentes de Educación física } \\
\hline & \multicolumn{2}{|c|}{$\begin{array}{l}\text { Principio } \\
\text { asignatura }\end{array}$} & \multicolumn{2}{|c|}{$\begin{array}{c}\text { Final } \\
\text { Asignatura }\end{array}$} \\
\hline & Media & DT & Media & DT \\
\hline $\begin{array}{l}\text { 3.1. Diseñar, aplicar y analizar intervenciones didácticas } \\
\text { en el área de Educación Física }\end{array}$ & 2.64 & .762 & 3 & .508 \\
\hline $\begin{array}{l}\text { 3.2. Diseñar, desarrollar y evaluar los procesos de } \\
\text { enseñanza-aprendizaje relativos a la actividad física y el } \\
\text { deporte con atención a las Necesidades Específicas de } \\
\text { Apoyo Educativo }\end{array}$ & 2.44 & .773 & 2.88 & .554 \\
\hline $\begin{array}{l}\text { 3.3. Conocer y promover las diferentes manifestaciones } \\
\text { motrices que forman parte de la cultura tradicional }\end{array}$ & 2.31 & .710 & 2.84 & 677 \\
\hline $\begin{array}{l}\text { 3.4. Conocer y aplicar los fundamentos biológicos, } \\
\text { fisiológicos, de maduración evolutiva y el desarrollo } \\
\text { psicomotor }\end{array}$ & 2.22 & .722 & 2.88 & .609 \\
\hline $\begin{array}{l}\text { 3.5. Diseñar, modificar y/o adaptar al contexto educativo } \\
\text { situaciones motrices orientadas al desarrollo y } \\
\text { perfeccionamiento de las habilidades motrices }\end{array}$ & 2.39 & .728 & 3.16 & .677 \\
\hline $\begin{array}{l}\text { 3.6. Conocer los elementos y fundamentos de la expresión } \\
\text { corporal y la comunicación no verbal y su valor formativo y } \\
\text { cultural }\end{array}$ & 2.61 & .728 & 3.28 & .581 \\
\hline $\begin{array}{l}\text { 3.7. Saber utilizar el juego como recurso didáctico y como } \\
\text { contenido de enseñanza }\end{array}$ & 3.33 & .632 & 3.44 & .619 \\
\hline $\begin{array}{l}\text { 3.8. Conocer los fundamentos básicos de la iniciación } \\
\text { deportiva escolar y diseñar tareas específicas para } \\
\text { utilizarlos en el ámbito de la enseñanza }\end{array}$ & 2.64 & .639 & 3.09 & .588 \\
\hline $\begin{array}{l}\text { 3.9. Identificar los riesgos que se derivan para la salud de } \\
\text { la práctica de actividades físicas inadecuadas }\end{array}$ & 2.86 & .683 & 3 & .762 \\
\hline $\begin{array}{l}\text { 3.10. Diseñar un plan de hábitos de vida saludable (higiene } \\
\text { y alimentación) y de práctica de actividad física regular. }\end{array}$ & 3.11 & .708 & 3.03 & .740 \\
\hline $\begin{array}{l}\text { 3.11. Saber aplicar los fundamentos (técnicas) de las } \\
\text { actividades físicas en el medio natural }\end{array}$ & 2.86 & .723 & 2.91 & .641 \\
\hline $\begin{array}{l}\text { 3.12. Saber utilizar diferentes instrumentos de evaluación } \\
\text { en el área de Educación Física }\end{array}$ & 2.49 & .702 & 3.19 & .592 \\
\hline $\begin{array}{l}\text { 3.13. Tener capacidad de reflexión sobre el proceso de } \\
\text { enseñanza/aprendizaje y las distintas metodologías dentro } \\
\text { de las clases de Educación Física }\end{array}$ & 2.61 & .688 & 3.16 & .583 \\
\hline $\begin{array}{l}\text { 3.14. Analizar y comunicar, de manera crítica y } \\
\text { fundamentada, el valor de la actividad física y el deporte y } \\
\text { sus posibilidades de contribuir al desarrollo y bienestar de } \\
\text { las personas }\end{array}$ & 2.86 & .723 & 3.13 & .660 \\
\hline
\end{tabular}

En este último bloque del cuestionario se puede observar como las diferencias entre la media aritmética al principio de la asignatura y al final de la asignatura han aumentando de forma considerable. Los ítems que más han aumentado su media han sido: diseñar, modificar y/o adaptar al contexto educativo situaciones motrices orientadas al desarrollo y perfeccionamiento de las habilidades motrices, conocer los elementos y fundamentos de la expresión corporal y la comunicación no verbal y su valor formativo y cultural, y saber utilizar diferentes instrumentos de evaluación en el área de Educación Física. 
El único ítem de este bloque que ha sufrido una bajada de la media aritmética al final de la asignatura ha sido diseñar un plan de hábitos de vida saludable (ver tabla 6).

\subsection{Rendimiento académico}

En este apartado vamos a analizar el rendimiento académico obtenido por el alumnado en la asignatura. La distribución del número de alumnos según la calificación se recoge en la tabla 7:

Tabla 7.

Resultados globales de calificación de la asignatura.

\begin{tabular}{lcc}
\hline \multicolumn{3}{c}{ Resultados globales } \\
\hline Calificación & Porcentaje & $N^{0}$ alumnos/as \\
\hline Matrícula Honor & --- & 0 \\
\hline Sobresaliente & --- & 0 \\
\hline Notable & $72.34 \%$ & 34 \\
\hline Aprobado & $12.76 \%$ & 6 \\
\hline Suspenso & $6.38 \%$ & 3 \\
\hline No presentado & $8.52 \%$ & 4 \\
\hline Totales & 100 & 47 \\
\hline
\end{tabular}

Como podemos observar, la mayoría del grupo se encuentra en un notable. Pero es importante señalar que no existe ningún sobresaliente en el grupo que estamos analizando. Esto se ha debido, al principio, al desconocimiento de este tipo de sistemas de evaluación por parte del alumnado del primer curso de grado, pero, en gran medida, a la falta de hábito de estudio y de trabajo, y al escaso interés y preocupación mostrada por corregir y mejorar sus trabajos cuando se les solicitaba. Aunque la mayoría hace lo necesario para aprobar. Solo tres alumnos suspenden la materia.

En la tabla 8 puede observarse las diferencias de rendimiento académico entre las diferentes vías de evaluación. También puede observarse que porcentaje de alumnado opta por cada vía de evaluación; concretamente, el 55,32\% sigue la vía continua, el $34,04 \%$ opta por la vía mixta y el $2,13 \%$ realiza la vía de examen final. Los no presentados son el $8,51 \%$.

Tabla 8.

Resultados del rendimiento académico de las distintas vías de evaluación.

\begin{tabular}{lccccccc}
\hline \multicolumn{1}{c}{ Vías } & NP & Suspenso & Aproba. & Notable & Sobresaliente & $\begin{array}{c}\text { Mat. } \\
\text { Honor }\end{array}$ & $\begin{array}{c}\text { Totalesl } \\
\text { vías }\end{array}$ \\
\hline Continua & - & - & - & 55.32 & - & - & $55.32 \%$ \\
\hline Mixta & - & 4.26 & 12.76 & 17.02 & - & - & $34.04 \%$ \\
\hline Examen & 8.51 & 2.13 & - & & - & - & $10.64 \%$ \\
\hline $\begin{array}{l}\text { Totales por } \\
\text { calificaciones }\end{array}$ & 8.51 & 6.39 & 12.76 & 72.34 & 0 & 0 & 100 \\
\hline
\end{tabular}




\subsection{Carga de trabajo}

En este apartado se revisa la carga de trabajo que supone la asignatura para el profesorado y para el alumnado.

\subsubsection{Carga de trabajo para el profesorado}

La asignatura cuenta con un total de 44 alumnos, con seis horas lectivas a la semana y seis horas semanales de tutorías. En la tabla 9 detallamos la carga de trabajo para el profesorado en horas mensuales.

Tabla 9.

Resultados de las horas mensuales de carga de trabajo del profesorado.

\begin{tabular}{|c|c|c|c|c|c|c|}
\hline & \multicolumn{6}{|c|}{ Asignaturas de un único cuatrimestre } \\
\hline & 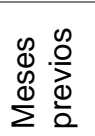 & $\begin{array}{l}\mathscr{\varrho} \\
\stackrel{\Xi}{E} \\
\circ \\
\stackrel{-}{1}\end{array}$ & $\begin{array}{l}\mathscr{E} \\
\stackrel{E}{E} \\
\stackrel{N}{N}\end{array}$ & $\begin{array}{l}\mathscr{\mathscr { E }} \\
\stackrel{E}{E} \\
\stackrel{m}{m}\end{array}$ & $\begin{array}{l}\stackrel{\mathscr{U}}{E} \\
\stackrel{\square}{\square}\end{array}$ & Totales \\
\hline $\mathrm{PC}=$ preparación de clases & 25 & 14 & 20 & 18 & 12 & 89 \\
\hline$C D=$ corrección de documentos & & 31 & 42 & 41 & 25 & 139 \\
\hline $\begin{array}{l}\text { MW }=\text { mantenimiento de web }+ \text { e- } \\
\text { mail con alumnado }\end{array}$ & 4 & 6 & 8 & 8 & 7 & 33 \\
\hline subtotales & 29 & 51 & 70 & 137 & 44 & 261 \\
\hline
\end{tabular}

Como puede apreciarse la carga de trabajo del profesorado es muy grande, especialmente en lo relativo a corrección de documentos, pero también en preparación de clases. La explicación más lógica es que este año la asignatura se ha tenido que impartir por un grupo de 4 profesores noveles, lo cual afecta a esos dos parámetros: la preparación de clases exige mucho más tiempo que cuando se tiene experiencia y, por otra parte, la menor práctica corrigiendo trabajos y documentos del alumnado hace que necesiten también más tiempo del habitual para esta actividad. Son dos aspectos en los que la carga de trabajo se rebaja notablemente cuanto mayor es la experiencia docente. Si tenemos en cuenta que se trata de una asignatura de 6 créditos, sale una media de 45 horas de carga extra de trabajo para el profesor por crédito, además de las horas lectivas.

\subsubsection{Carga de trabajo para el alumnado}

En la tabla 10 se analiza la carga de trabajo que supone para el alumnado, organizado por horas mensuales que han dedicado a cada tarea realizada en la asignatura. Estos datos se han recogido a través de un cuestionario específico sobre documentos elaborados y actividades de aprendizaje realizadas.

Tabla 10.

Resultados de las horas mensuales de carga de trabajo del alumnado.

\begin{tabular}{lcccccc}
\hline \multicolumn{1}{c}{ Tiempo de trabajo que ha supuesto } & $\begin{array}{l}\mathbf{1}^{\text {er }} \\
\text { mes }\end{array}$ & $\begin{array}{l}\mathbf{2}^{\mathbf{0}} \\
\text { mes }\end{array}$ & $\begin{array}{l}\mathbf{3}^{\mathbf{0}} \\
\text { mes }\end{array}$ & $\begin{array}{l}\mathbf{4}^{\mathbf{0}} \\
\text { mes }\end{array}$ & $\begin{array}{l}\text { TOTALES } \\
\text { PARCIALES }\end{array}$ \\
\hline Lecturas y recensiones. & & 5 & 10 & 5 & 20 \\
\hline Preparación y/o informes de sesiones prácticas. & 8 & 8 & 6 & 6 & 28 \\
\hline Trabajos e informes de temas. & 10 & 8 & - & - & 18 \\
\hline $\begin{array}{l}\text { Revisiones y reconducciones de los trabajos } \\
\text { (grupal y en tutoría). }\end{array}$ & 5 & 10 & 5 & & 20 \\
\hline Estudiar para el examen & - & & 5 & 12 & 17 \\
\hline Subtotales por meses & 23 & 31 & 26 & 23 & 98 \\
\hline
\end{tabular}


En este caso se observa que la carga media total de trabajo no presencial es de 98 horas. A esto habría que sumar la carga lectiva presencial, que es de 60 horas, lo que nos da un total de 158 horas, incluidas horas presenciales y no presenciales. Dado que la asignatura es de 6 créditos ECTS, la carga total debería ser de 150 horas. Teniendo esto en cuenta, podemos comprobar que la percepción del alumnado de que trabajan mucho, mucho más de lo que deberían, no se ajusta a lo establecido legalmente. Realmente, la media de trabajo extra es mínima.

En la tabla 11 se analiza si existe diferencia en la carga de trabajo del alumnado en función de la calificación obtenida, así como el máximo y el mínimo.

Tabla 11.

Resultados de la carga de trabajo en función de la calificación obtenida.

\begin{tabular}{|c|c|c|c|c|c|c|}
\hline \multirow{2}{*}{$\begin{array}{c}\text { Carga media de } \\
\text { trabajo autónomo } \\
\text { para el alumnado a } \\
\text { lo largo del } \\
\text { cuatrimestre, } \\
\text { aparte de las horas } \\
\text { lectivas }\end{array}$} & \multirow{2}{*}{$\begin{array}{l}\text { Carga del alumno-a } \\
\text { que menos y que } \\
\text { más ha hecho en el } \\
\text { grupo (en horas) }\end{array}$} & \multicolumn{5}{|c|}{$\begin{array}{l}\text { Medias de carga de trabajo (horas) en } \\
\text { función de la calificación del alumnado }\end{array}$} \\
\hline & & $\begin{array}{l}\text { Sus- } \\
\text { penso }\end{array}$ & $\begin{array}{c}\text { Sufi- } \\
\text { ciente }\end{array}$ & $\begin{array}{l}\text { Nota } \\
\text {-ble }\end{array}$ & $\begin{array}{l}\text { Sobresa } \\
\text {-liente }\end{array}$ & $\begin{array}{l}\text { Matrícul } \\
\text { a Honor }\end{array}$ \\
\hline 98 horas & $\begin{array}{l}\text { valor mínimo: } 19 \\
\text { valor máximo: } 145\end{array}$ & 56 & 75 & 101 & & \\
\hline
\end{tabular}

Como podemos observar en la tabla 11, la carga de trabajo en el alumnado que ha obtenido la calificación de notable ha sido una media de de 101 horas. Dado que la asignatura es de 6 créditos ECTS, en nuestra facultad eso corresponde a 60 horas presenciales y 90 no presenciales. Por tanto, la carga de trabajo media es ligeramente superior a lo establecido teóricamente. Sólo los alumnos de calificaciones más bajas están por debajo de dicha carga, mientras que el alumnado de notable está ligeramente por encima.

\section{CONCLUSIONES}

En general la experiencia ha sido muy positiva, tanto para el alumnado como para el profesorado. La idea es mantener la práctica de éxito y volver a utilizar de forma sistemática la ficha detallada de autoevaluación y autocalificación (escala descriptiva, elaborada por López et al. (2016).

Como puede apreciarse la carga de trabajo del profesorado se ha incrementado notablemente respecto a otros años. La única explicación que encontramos es que este año la asignatura se ha tenido que impartir por un grupo de 4 profesores noveles, lo cual afecta a esos parámetros, tanto porque la preparación de clases existe más tiempo, como porque la menor práctica corrigiendo trabajos y documentos del alumnado hace que necesiten mucho más tiempo para esta actividad. Son dos aspectos en los que la carga de trabajo se suele rebajar notablemente cuanto mayor es la experiencia docente.

En cuanto al alumnado, podemos observar cómo los que han obtenido buenas calificaciones sí que han dedicado suficientes horas al trabajo de la asignatura, pero no lo suficiente como para sacar las calificaciones más altas. En comparación a promociones anteriores, se observa un menor interés y capacidad de trabajo por parte del alumnado. 


\section{REFERENCIAS}

Barba Martín, J., López Pastor, V. M., Manrique Arribas, J.C., Gea Fernández, J.M., \& Monjas Aguado, R. (2010) Garantir l'èxit en la formació inicial del professorat d'educació física: els projectes d'aprenentatge tutelats. Temps d'educació, 39, 187-206.

Castejón-Oliva, F., Santos-Pastor, M., \& Palacios, A. (2015). Cuestionario sobre metodología y evaluación en formación inicial en educación física. Revista Internacional de Medicina y Ciencias de la Actividad Física y del Deporte, 15 (58), 245-267. Recuperado de http://cdeporte.rediris.es/revista/revista58/artescala566.htm

López-Pastor, V.M., Manrique Arribas, J. C., Monjas Aguado, R., \& Gea Fernández, J. M. (2010). Formative Assessment in project-oriented learning to improve academic performance. Assessment, Learning \& Teaching Journal, 9, 23-26.

López, V.M., Castejón, J., \& Pérez, Á. (2012). ¿Implicar al alumnado en la evaluación en la formación inicial del profesorado? Un estudio de caso de evaluación entre iguales de un examen. Multidisciplinary Journal of Educational Research, 2(2), 177-201. DOI: 10.4471/remie.2012.09. (http://dx.doi.org/10.4471/remie.2012.09).

López-Pastor, V. M., Pérez-Pueyo, A., Barba Martín, J. J., \& Lorente, E. (2016) Rubricas y PATS. Utilización de una escala graduada (rubrica) para la autoevaluación de trabajos escritos en la formación inicial del profesorado de educación física (FIPEF). Percepción de su funcionalidad por parte de los estudiantes. Cultura, Ciencia, Deporte (CCD), 31 (11,1),37-49. DOI: $10.12800 / \mathrm{ccd}$

Manrique Arribas, J.C., López Pastor, V.M., Monjas Aguado, R., \& Real Rubio, F. (2010). El potencial de los proyectos de aprendizaje tutorado y los sistemas de evaluación formativa en la mejora de la autonomía del alumnado. Una experiencia interdisciplinar en formación inicial del profesorado. Revista Española de Educación Física y Deportes, 14, 39-57.

Romero-Martín, R., Fraile-Aranda, A., López-Pastor, V. M., \& Castejón-Oliva, F. J. (2014). Relación entre sistemas de evaluación formativa, rendimiento académico y carga de trabajo del profesor y del alumno en la docencia universitaria. Revista Infancia y Aprendizaje, 37(1), 16-32.

Salcines, I., González-Fernández, N., Ramírez-García, A., \& Martínez-Mínguez, L. (2018). Validación de la Escala de Autopercepción de Competencias Transversales y Profesionales de Estudiantes de Educación Superior. Profesorado. Revista de Currículum y Formación de Profesorado, 22(3), 31-51. 